\title{
ON $\lambda$-CONNECTIONS ON A CURVE WHERE $\lambda$ IS A FORMAL PARAMETER
}

\author{
D. ARINKIN
}

\begin{abstract}
We study $\mathrm{GL}_{2}$-bundles with connections with a small parameter on a smooth projective curve. We describe an open subset in the moduli space of such bundles. The description degenerates into the Hitchin fibration as the parameter tends to zero.
\end{abstract}

\section{Introduction}

1.1. The moduli space of Higgs bundles on a curve admits a well-known description in terms of spectral curves (the Hitchin fibration). On the other hand, Higgs bundles can be viewed as a degeneration of bundles with connections: P. Deligne introduced the notion of ' $\lambda$-connections', and Higgs fields (resp. connections) are $\lambda$-connections for $\lambda=0$ (resp. $\lambda=1$ ). It is natural to ask whether spectral curves can be used to describe the moduli space of $\lambda$-connections for $\lambda \neq 0$.

The simplest case is when $\lambda \in \mathbb{C}[[\lambda]]$ is a formal parameter; that is, the $\lambda$ connections considered are formal deformations of Higgs bundles. This case has the following advantage: if a $\lambda$-connection is a formal deformation of a Higgs bundle, we can try using the spectral curve corresponding to the Higgs bundle to describe the $\lambda$-connection. Informally, if $\lambda$ is an actual number rather than a formal parameter (for instance, $\lambda=1$ ), we would not know which spectral curve to use.

Let Conn $_{\lambda}$ be the moduli space of $\lambda$-connections: Conn $_{\lambda}$ parametrizes triples $(L, \nabla, \lambda)$, where $L$ is a $G$-bundle on $X, \lambda \in \mathbb{C}$, and $\nabla$ is a $\lambda$-connection on $L$. Here $X$ is a smooth curve and $G$ is a reductive group. The moduli stack of Higgs bundles is the closed substack Higgs $\subset \mathbf{C o n n}_{\lambda}$ given by $\lambda=0$. Making $\lambda$ a formal parameter corresponds to working with the formal completion $\operatorname{Conn}_{\text {form }}$ of $\mathbf{C o n n}_{\lambda}$ along Higgs instead of $\mathbf{C o n n}_{\lambda}$ itself. The problem simplifies further if we consider only those Higgs bundles that are non-degenerate in some sense. Geometrically, this corresponds to taking an open substack Higgs ${ }^{\prime} \subset$ Higgs (parametrizing non-degenerate bundles) and studying the formal completion $\mathbf{C o n n}_{\text {form }}^{\prime}$ of Conn $_{\lambda}$ along Higgs'.

Received by the editors November 2, 2004.

Partially supported by NSF grants DMS-0100108 and DMS-0401164 and by DARPA grant HR0011-04-1-0031. 
In this paper, we set $G=\mathrm{GL}_{2}$ and consider Higgs bundles whose spectral curves are smooth, but possibly ramified. We use spectral curves to describe $\lambda$-connections that are formal deformations of such Higgs bundles (Theorem A), and then derive a description of $\mathbf{C o n n}_{\text {form }}^{\prime}$ if $X$ is projective (Theorem B). The results can be generalized to arbitrary reductive group (see Section 2.3). An expanded version of this paper is available online [1].

Remark. Although $\lambda$-connections are interesting geometric objects in their own right, they are particularly important because they can be used to compactify the moduli stack Conn of ordinary connections ([6], [7]). We hope that studying Conn $_{\text {form }}$ can improve our understanding of Conn (which is important, for instance, in the geometric Langlands program). One case $\left(\mathrm{SL}_{2}\right.$-connections on $\mathbb{P}^{\mathbf{1}}$ with four simple poles) appears in [2]: a result about Conn form $_{\text {( }}$ ([2, Proposition $6]$ ) is used to compute the cohomology groups $H^{i}(\mathbf{C o n n}, F)$ for some natural coherent sheaves $F$.

1.2. Conventions and notation. In this work, the ground field is $\mathbb{C}$, that is, 'scheme' means ' $\mathbb{C}$-scheme', $\mathrm{GL}_{2}$ means $\mathrm{GL}_{2}(\mathbb{C})$, and so on. However, our methods are purely algebraic, so $\mathbb{C}$ can be replaced by any algebraically closed field of characteristic zero.

For a scheme (or a formal scheme, or a stack) $S$, the words 'groupoid of vector bundles on $S^{\prime}$ refer to the category whose objects are vector bundles on $S$ and whose arrows are isomorphisms of vector bundles. The same convention applies to vector bundles with additional structures (e.g., connections).

\section{Main results}

2.1. Let $X$ be a smooth (not necessarily projective) curve over $\mathbb{C}$. Our first result describes $\mathbb{C}[[\lambda]]$-families of $\lambda$-connections on $X$ using spectral curves.

Definition 2.1. Let $L$ be a vector bundle on $X, \lambda \in \mathbb{C}$. A $\lambda$-connection on $L$ is a $\mathbb{C}$-linear map $\nabla: L \rightarrow L \otimes \Omega_{X}$ such that

$$
\nabla(f s)=f \nabla(s)+\lambda s \otimes d f
$$

for any $f \in O_{X}, s \in L$. A Higgs field is a $\lambda$-connection for $\lambda=0$.

Definition 2.2. A $\mathbb{C}[[\lambda]]$-family of vector bundles with $\lambda$-connections on $X$ is a pair $(L, \nabla)$, where $L$ is a vector bundle on the formal scheme $X[[\lambda]]:=\lim _{\longrightarrow} X \times$ Spec $\mathbb{C}[\lambda] /\left(\lambda^{i}\right)$, and $\nabla: L \rightarrow L \otimes_{O_{X}} \Omega_{X}$ is a $\mathbb{C}[[\lambda]]$-linear $\lambda$-connection.

The reduction of $(L, \nabla)$ modulo $\lambda$ is the Higgs bundle (that is, a bundle equipped with a Higgs field) $\left(L_{0}, \nabla_{0}\right)$ on $X$, where $L_{0}:=L / \lambda L$ is the restriction of $L$ to $X$, and $\nabla_{0}: L_{0} \rightarrow L_{0} \otimes \Omega_{X}$ is induced by $\nabla$.

Definition 2.3. A subscheme $\tilde{X} \subset T^{*} X$ is a spectral curve (for $\mathrm{GL}_{2}$ ) if the projection $p_{\tilde{X}}:=\left.p\right|_{\tilde{X}}: \tilde{X} \rightarrow X$ is flat and finite of degree 2 . Here $p: T^{*} X \rightarrow$ $X$ is the cotangent bundle. The natural 1-form $\mu=\mu_{\tilde{X}} \in H^{0}\left(\tilde{X}, p_{\tilde{X}}^{*} \Omega_{X}\right) \subset$ $H^{0}\left(\tilde{X}, \Omega_{\tilde{X}}\right)$ is the restriction of the natural 1-form $\mu_{T^{*} X} \in H^{0}\left(T^{*} X, p^{*} \Omega_{X}\right)$. 
Fix a smooth spectral curve $\tilde{X} \subset T^{*} X$. Denote by $\mathcal{C}_{o n n}(\tilde{X})$ the groupoid of $\mathbb{C}[[\lambda]]$-families $(L, \nabla)$ of rank 2 bundles with $\lambda$-connections on $X$ such that $\tilde{X}$ equals the spectral curve of $\left(L_{0}, \nabla_{0}\right)$, where $\left(L_{0}, \nabla_{0}\right)$ is the reduction of $(L, \nabla)$ modulo $\lambda$. (The notion of the spectral curve of a Higgs bundle is recalled in Theorem 5.1.)

Denote by $\widetilde{\mathcal{C o n n}}{ }_{\lambda}(\tilde{X})$ the groupoid of $\mathbb{C}[[\lambda]]$-families $(l, \delta)$ of rank 1 bundles with $\lambda$-connections on $\tilde{X}$ such that $\delta: l \rightarrow l \otimes \Omega_{\tilde{X}}\left(\tilde{x}_{1}+\cdots+\tilde{x}_{n}\right)$ has first order poles at $\tilde{x}_{1}, \ldots, \tilde{x}_{n}$ (the ramification locus of $p_{\tilde{X}}: \tilde{X} \rightarrow X$ ), the residue of $\delta$ at $\tilde{x}_{i}$ equals $-\lambda / 2$ (the notion of residue of a $\lambda$-connection is straightforward), and the reduction $\delta_{0}$ of $\delta$ modulo $\lambda$ equals $\mu_{\tilde{X}}$. Notice that $\delta_{0}$ is a Higgs field on the line bundle $l / \lambda l$, and a Higgs field on a line bundle is simply a 1 -form.

The following theorem goes back to $\mathrm{W}$. Wasow; it is a slightly generalized version of [4, Proposition 1.2] (see also [8, Theorem 25.2]) and can be proved by the same method:

Theorem 2.4. Suppose $X$ is a smooth curve and $p_{\tilde{X}}: \tilde{X} \rightarrow X$ is an unramified spectral curve. The functor $\widetilde{\mathcal{C o n n}} n_{\lambda}(\tilde{X}) \rightarrow \mathcal{C o n n}_{\lambda}(\tilde{X})$ that sends $(l, \delta)$ to $\left(\left(p_{\tilde{X}}\right)_{*}(l),\left(p_{\tilde{X}}\right)_{*}(\delta)\right)$ is an equivalence of groupoids.

Let now $\tilde{X}$ be a smooth (but possibly ramified) spectral curve. (Notice that $\tilde{X}$ is always ramified if $X$ is a projective curve of genus at least 2.) We claim that the groupoids $\widetilde{\mathcal{C o n n}}(\tilde{X})$ and $\mathcal{C o n n}_{\lambda}(\tilde{X})$ are still equivalent in this case; however, the equivalence is not as explicit as that in Theorem 2.4.

Set $\tilde{X}_{u}:=\tilde{X}-\left\{\tilde{x}_{1}, \ldots, \tilde{x}_{n}\right\}$, where $\tilde{x}_{i} \in \tilde{X}$ are the ramification points of $\tilde{X} \rightarrow X$, and $X_{u}:=p_{\tilde{X}}\left(\tilde{X}_{u}\right) \subset X$. There are natural functors $\mathcal{C}_{\text {onn }}(\tilde{X}) \rightarrow$ $\operatorname{Conn}_{\lambda}\left(\tilde{X}_{u}\right)$ (restriction to $\left.X_{u} \subset X\right)$ and $\widetilde{\mathcal{C o n n}}{ }_{\lambda}(\tilde{X}) \rightarrow \widetilde{\mathcal{C o n n}} \widetilde{X}_{\lambda}\left(\tilde{X}_{u}\right)$ (restriction to $\left.\tilde{X}_{u} \subset \tilde{X}\right)$.

Theorem A. Let $X$ be a smooth curve and $\tilde{X} \subset T^{*} X$ a smooth spectral curve.

1. The functor $\mathcal{C o n n}_{\lambda}(\tilde{X}) \rightarrow \mathcal{C o n n}_{\lambda}\left(\tilde{X}_{u}\right)$ is fully faithful (so that $\mathcal{C} o n n_{\lambda}(\tilde{X})$ is equivalent to a full subcategory of $\left.\mathcal{C o n n}_{\lambda}\left(\tilde{X}_{u}\right)\right)$.

2. The functor $\widetilde{\mathcal{C o n n}}{ }_{\lambda}(\tilde{X}) \rightarrow \widetilde{\mathcal{C o n n}}_{\lambda}\left(\tilde{X}_{u}\right)$ is fully faithful.

3. For a groupoid $\mathcal{G}$, let $[\mathcal{G}]$ be the set of isomorphism classes of objects of $\mathcal{G}$. Note that Theorem 2.4 gives an equivalence $\widetilde{\mathcal{C o n n}}{ }_{\lambda}\left(\tilde{X}_{u}\right) \widetilde{\rightarrow} \mathcal{C o n n}{ }_{\lambda}\left(\tilde{X}_{u}\right)$, which induces an isomorphism $\left[\widetilde{\mathcal{C o n n}}_{\lambda}\left(\tilde{X}_{u}\right)\right] \widetilde{\mathcal{G}}\left[\operatorname{Conn}_{\lambda}\left(\tilde{X}_{u}\right)\right]$. We claim that the isomorphism identifies $\left[\widetilde{\mathcal{C o n n}}_{\lambda}(\tilde{X})\right] \subset\left[\widetilde{\mathcal{C o n n}}_{\lambda}\left(\tilde{X}_{u}\right)\right]$ and $\left[\mathcal{C o n n}{ }_{\lambda}(\tilde{X})\right] \subset$ $\left[\operatorname{Conn}_{\lambda}\left(\tilde{X}_{u}\right)\right]$.

Corollary 2.5. In the assumptions of Theorem A, there is an equivalence of groupoids $\mathcal{F}: \widetilde{\mathcal{C o n n}}_{\lambda}(\tilde{X}) \widetilde{\rightarrow} \operatorname{Conn}_{\lambda}(\tilde{X})$ that makes the diagram

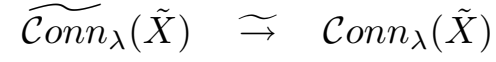

$$
\begin{aligned}
& \widetilde{\mathcal{C o n n}}_{\lambda}\left(\tilde{X}_{u}\right) \stackrel{\downarrow}{\downarrow} \operatorname{Conn}_{\lambda}\left(\tilde{X}_{u}\right)
\end{aligned}
$$


commute (by definition, this means that there exists an isomorphism $\phi$ between the two composition functors given by the diagram). The pair $(\mathcal{F}, \phi)$ is unique up to a canonical isomorphism.

We prove Theorem A by direct calculations: we first reduce $\lambda$-connections to a standard form, and then construct $\mathcal{F}$ essentially by writing formal solutions to the corresponding differential equation. Another (slightly longer, but less calculative) proof of the theorem is presented in [1].

Remark. $\widetilde{\mathcal{C o n n}}{ }_{\lambda}(\tilde{X})$ has a simpler description. For any $(l, \delta) \in \widetilde{\mathcal{C o n n}}(\tilde{X})$, define a connection $\partial: l \rightarrow l \otimes \Omega_{\tilde{X}}\left(\tilde{x}_{1}+\cdots+\tilde{x}_{n}\right)$ by $\partial:=\lambda^{-1}(\delta-\mu)$. In this way, $\widetilde{\mathcal{C o n n}}_{\lambda}(\tilde{X})$ is identified with the groupoid of pairs $(l, \partial)$, where $l$ is a line bundle on $\tilde{X}[[\lambda]]$, and $\partial: l \rightarrow l \otimes \Omega_{\tilde{X}}\left(\tilde{x}_{1}+\cdots+\tilde{x}_{n}\right)$ is a $(\mathbb{C}[[\lambda]]$-linear $)$ connection whose residues at $\tilde{x}_{i} \in \tilde{X}$ equal $-1 / 2$.

2.2. Suppose now that the smooth curve $X$ is projective. Denote by Higgs the moduli stack of rank 2 Higgs bundles $(L, \nabla)$ on $X$. Let $\mathbf{C o n n}_{\lambda}$ be the moduli stack of triples $(L, \nabla, \lambda)$, where $\lambda \in \mathbb{C}, L$ is a rank 2 bundle on $X$ and $\nabla$ is a $\lambda$-connection on $L$. Then Higgs is identified with the closed substack of $\mathbf{C o n n}_{\lambda}$ formed by triples $(L, \nabla, \lambda)$ with $\lambda=0$. Our next result describes an open subset in the formal completion of $\mathbf{C o n n}_{\lambda}$ along Higgs.

Denote by SCurv the space of spectral curves $\tilde{X}$. It is isomorphic to an affine space: the coordinates on SCurv are the coefficients of the equation for $\tilde{X}$. Denote by $p_{H}:$ Higgs $\rightarrow$ SCurv the morphism that sends a Higgs bundle to its spectral curve (the Hitchin fibration). The fiber of $p_{H}$ over a smooth spectral curve $\tilde{X} \in$ SCurv is the moduli stack of line bundles on $\tilde{X}$.

Denote by $\mathbf{M}^{\sharp}$ the moduli stack of collections $(\tilde{X}, l, \partial)$, where $\tilde{X} \in$ SCurv is a smooth spectral curve, $l$ is a line bundle on $\tilde{X}$, and $\partial: l \rightarrow l \otimes \Omega_{\tilde{X}}\left(\tilde{x}_{1}+\cdots+\tilde{x}_{n}\right)$ is a connection (not a $\lambda$-connection) whose residues at $\tilde{x}_{1}, \ldots, \tilde{x}_{n}$ equal $-1 / 2$. As before, $\tilde{x}_{1}, \ldots, \tilde{x}_{n}$ are the ramification points of $p_{\tilde{X}}: \tilde{X} \rightarrow X$.

Consider the projection

$$
p^{\sharp}: \mathbf{M}^{\sharp} \rightarrow \text { Higgs : }(\tilde{X}, l, \delta) \mapsto(\tilde{X}, l) ;
$$

here we identify Higgs bundles with their spectral data $(\tilde{X}, l)$ (see Theorem 5.1). The fiber of $p^{\sharp}$ over $(\tilde{X}, l)$ is the space of connections $\partial: l \rightarrow l \otimes \Omega_{\tilde{X}}\left(\tilde{x}_{1}+\cdots+\tilde{x}_{n}\right)$, $\operatorname{res}_{\tilde{x}_{i}} \partial=-1 / 2$. The following statement is immediate:

Lemma 2.6. Set Higgs' $:=p^{\sharp}\left(\mathbf{M}^{\sharp}\right) \subset$ Higgs.

1. Higgs' $\subset$ Higgs is an open substack.

2. $(L, \nabla) \in$ Higgs' $^{\prime}$ if and only if the spectral curve $\tilde{X} \subset T^{*} X$ of $(L, \nabla)$ is smooth and for any connected component $X^{\prime} \subset X$ and $a \nabla$-invariant subbundle $\left.L^{\prime} \subset L\right|_{X^{\prime}}$, we have $\operatorname{deg}\left(L^{\prime}\right)=0$. (If $X$ is a connected curve of genus at least 2 , the second condition is equivalent to the condition $\operatorname{deg}(L)=0$.) 
3. The fiber $p^{\sharp-1}(L, \nabla)$ over $(L, \nabla) \in \mathbf{H i g g s}^{\prime}$ is an affine space; the corresponding vector space is $H^{0}\left(\tilde{X}, \Omega_{\tilde{X}}\right)$. More precisely: as $(L, \nabla)$ varies, the spaces $H^{0}\left(\tilde{X}, \Omega_{\tilde{X}}\right)$ form a vector bundle on Higgs ${ }^{\prime}$, and $p^{\sharp}: \mathbf{M}^{\sharp} \rightarrow$ Higgs $^{\prime}$ is a torsor over this vector bundle.

Denote by $\zeta_{0}$ the relative tangent bundle to $p^{\sharp}$; it is a foliation on $\mathbf{M}^{\sharp}$, and Higgs ${ }^{\prime}$ is identified with the quotient of $\mathbf{M}^{\sharp}$ modulo $\zeta_{0}$.

Remark. $\mathbf{M}^{\sharp}$ is an algebraic stack, so the notion of a foliation on $\mathbf{M}^{\sharp}$ requires clarification. However, the stack structure on $\mathbf{M}^{\sharp}$ (and on Higgs' ${ }^{\prime}$ ) is rather simple: the automorphism groups of all points are isomorphic, and $\mathbf{M}^{\sharp}$ is a gerbe over the corresponding coarse moduli space, $M^{\sharp}$. (If $X$ is a connected curve of genus at least 2 , then $\mathbf{M}^{\sharp} \rightarrow M^{\sharp}$ is a $\mathbf{G}_{\mathbf{m}}$-gerbe.) If we work with $M^{\sharp}$ instead of $\mathbf{M}^{\sharp}$, then $\zeta_{0}$ becomes a foliation on a smooth algebraic space; such objects are easy to define. The downside is that in this way we get a description of the coarse moduli space of $\lambda$-connections rather than the moduli stack. We could avoid this difficulty if we rigidify the moduli problem, for instance, by adding framings of vector bundles at some points.

On the other hand, it is not hard to define the notion of a foliation on an algebraic stack. From now on, we will ignore this difficulty and freely use foliations on $\mathbf{M}^{\sharp}$.

Let us construct another foliation $\zeta_{\infty}$ on $\mathbf{M}^{\sharp}$ using isomonodromic deformation. Consider the composition $p_{H} \circ p^{\sharp}: \mathbf{M}^{\sharp} \rightarrow$ SCurv. The fiber of $p_{H} \circ p^{\sharp}$ over a smooth spectral curve $\tilde{X} \in$ SCurv is canonically identified with fibers over infinitesimally close spectral curves (the fiber is essentially the space of rank 1 local systems on $\tilde{X}$ with monodromy -1 around the ramification points; therefore, the fiber does not change under deformations of $\tilde{X}$ ). More precisely, the morphism $p_{H} \circ p^{\sharp}: \mathbf{M}^{\sharp} \rightarrow$ SCurv carries a connection. Let $\zeta_{\infty}$ be the foliation of horizontal vector fields with respect to this connection.

Now consider $\zeta_{0}$ and $\zeta_{\infty}$ as abstract vector bundles rather than foliations. Over a point $(\tilde{X}, l, \partial) \in \mathbf{M}^{\sharp}$, the fiber of $\zeta_{0}$ equals $H^{0}\left(\tilde{X}, \Omega_{\tilde{X}}\right)$, while the fiber of $\zeta_{\infty}$ equals $H^{0}\left(\tilde{X}, N_{\tilde{X}}\right)$, where $N_{\tilde{X}}$ is the normal bundle to $\tilde{X} \subset T^{*} X$. The symplectic structure on $T^{*} X$ identifies $N_{\tilde{X}}$ with $\Omega_{\tilde{X}}$; therefore, the vector bundles $\zeta_{0}$ and $\zeta_{\infty}$ are isomorphic.

Remark 2.7. We choose the isomorphism $\Omega_{\tilde{X}} \widetilde{\rightarrow} N_{\tilde{X}}$ so that the diagram

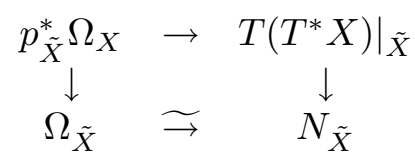

commutes. Here $\left.T\left(T^{*} X\right)\right|_{\tilde{X}}$ is the restriction to $\tilde{X} \subset T^{*} X$ of the tangent bundle to $T^{*} X$, the map $\left.p_{\tilde{X}}^{*} \Omega_{X} \rightarrow T\left(T^{*} X\right)\right|_{\tilde{X}}$ identifies $p_{\tilde{X}}^{*} \Omega$ with the subbundle of vertical vector fields, $p_{\tilde{X}}^{*} \Omega_{X} \rightarrow \Omega_{\tilde{X}}$ is the pull-back map for differential forms, and $\left.T\left(T^{*} X\right)\right|_{\tilde{X}} \rightarrow N_{\tilde{X}}$ is the natural projection. 
Definition 2.8. Let $\zeta_{0}, \zeta_{\infty} \subset T M$ be distributions on a smooth variety $M$, and let $\nu: \zeta_{0} \widetilde{\rightarrow} \zeta_{\infty}$ be an isomorphism of vector bundles on $M$. For $\alpha, \beta \in \mathbb{C}$, the linear combination $\alpha \zeta_{0}+\beta \zeta_{\infty} \subset T M$ is the image of the morphism $\alpha\left(\operatorname{id}_{\zeta_{0}}\right)+\beta \nu$ : $\zeta_{0} \rightarrow T M$ (in particular, if the morphism is an embedding of vector bundles, $\alpha \zeta_{0}+\beta \zeta_{\infty}$ is a distribution on $\left.M\right)$.

Theorem B. Let $\mathbf{M}^{\sharp}, \zeta_{0}$, and $\zeta_{\infty}$ be as above, and let us use the isomorphism $\zeta_{0} \widetilde{\rightarrow} \zeta_{\infty}$ from Remark 2.7 to construct the linear combination $\zeta_{\lambda}:=\zeta_{0}-\lambda \zeta_{\infty}$, $\lambda \in \mathbb{C}$. Notice that $\zeta_{0}$ and $\zeta_{\infty}$ are transversal, so $\zeta_{\lambda}$ is a distribution for any $\lambda \in \mathbb{C}$.

1. $\zeta_{\lambda}$ is a foliation on $\mathbf{M}^{\sharp}$ for any $\lambda \in \mathbb{C}$.

2. The quotient $\mathbf{M}^{\sharp} / \zeta_{\lambda}$ exists if $\lambda \in \mathbb{C}[[\lambda]]$ is a formal parameter, and such quotients form a family $\mathbf{M}^{\sharp}[[\lambda]] / \zeta_{\lambda} \rightarrow \operatorname{Spf} \mathbb{C}[[\lambda]]$ over the formal disc.

3. $\mathbf{M}^{\sharp}[[\lambda]] / \zeta_{\lambda}$ is canonically isomorphic to the formal completion of $\mathbf{C o n n}_{\lambda}$ along Higgs'. This isomorphism respects the projection to $\operatorname{Spf} \mathbb{C}[[\lambda]]$ (intuitively, $\mathbf{M}^{\sharp} / \zeta_{\lambda}$ is identified with an open substack in the moduli stack of $\lambda$-connections when $\lambda$ is a formal parameter).

2.3. Our results still hold for bundles over an arbitrary reductive group $G$. Let us sketch the generalization (the details will be given elsewhere). Let $\mathfrak{g}$ be the Lie algebra of $G, \mathfrak{h}$ its Cartan algebra, and $W$ the Weyl group. Recall ([3]) that to a Higgs bundle over $G$ on a smooth curve $X$ there corresponds a cameral cover $X_{\text {cam }} \rightarrow X$ : locally on $X$, a Higgs field is essentially a map $X \rightarrow \mathfrak{g}$, and $X_{\text {cam }}$ is given by $\mathfrak{h} \times_{\mathfrak{h} / W} X$, where the map $X \rightarrow \mathfrak{h} / W$ is the composition

$$
X \rightarrow \mathfrak{g} \rightarrow \mathfrak{g} / G=\mathfrak{h} / W .
$$

$X_{\text {cam }}$ is a closed subscheme of $T^{*} X \otimes \mathfrak{h}$ (the total space of the bundle $\Omega_{X} \otimes_{\mathbb{C}} \mathfrak{h}$ ), and the action of $W$ on $T^{*} X \otimes \mathfrak{h}$ preserves $X_{\text {cam }}$.

For a given cameral cover $X_{\text {cam }} \subset T^{*} X \otimes \mathfrak{h}$, denote by $\mathcal{C}_{0} n_{\lambda}\left(X_{\text {cam }}\right)$ the groupoid of $\mathbb{C}[[\lambda]]$-families of $G$-bundles with $\lambda$-connections on $X$ whose reductions modulo $\lambda$ are Higgs bundles with cameral cover $X_{\text {cam }}$. The generalization of Theorem A provides a description of $\mathcal{C o n n}_{\lambda}\left(X_{\text {cam }}\right)$ in terms of line bundles with $\lambda$-connections on $X_{\text {cam }}$ provided $X_{\text {cam }}$ is smooth. The proof is based on the following simple observation:

Lemma 2.9. Suppose $X_{\text {cam }} \subset T^{*} X \otimes \mathfrak{h}$ is a smooth cameral curve, $(L, \nabla) \in$ $\mathcal{C}$ onn $_{\lambda}\left(X_{\text {cam }}\right)$, and $x \in X$. Then in the formal neighborhood of $x$, the $\mathbb{C}[[\lambda]]$ family of $\lambda$-connections $(L, \nabla)$ is induced from $G^{\prime} \subset G$. Here $G^{\prime}$ is a reductive group that depends on $X_{\text {cam }}$ and $x$ only. $G^{\prime}$ is a torus if $X_{\text {cam }} \rightarrow X$ is unramified at $x$; $G^{\prime}$ has semisimple rank 1 (that is, $G^{\prime}$ is an extension of $S L_{2}$ or $P G L_{2}$ by a torus) if $X_{\text {cam }} \rightarrow X$ is ramified at $x$.

It follows from Lemma 2.9 that the 'formal' statement (Theorem 3.1) for GL 2 implies the corresponding theorem for $G$, which in turn implies the generalized Theorem A. We can then derive an analogue of Theorem B. 
2.4. Organization. In Section 3, we prove Theorem A by deriving it from its 'formal' version (Theorem 3.1), in which $X$ is a formal disc rather than a curve. We prove Theorem B in Section 4. In Section 5, we explain the relation between Theorem A and the description of Higgs bundle via spectral curves (which is reminded in Theorem 5.1). Finally, in Section 6, we sketch a geometric construction which generalizes the construction of Theorem B.

\section{Proof of Theorem A}

3.1. All of the above definitions ( $\lambda$-connections, Higgs bundles, spectral curves, etc.) still make sense if $X$ is a formal disc rather than a smooth curve. Therefore, we can formulate a 'formal' version of Theorem A:

Theorem 3.1. Let $X \simeq \operatorname{Spf} \mathbb{C}[[z]]$ be a formal disc and $\tilde{X} \subset T^{*} X$ a smooth spectral curve. Then statements (1)-(3) of Theorem A hold.

Theorem 3.1 implies Theorem A:

Proof of Theorem $A$. Let $X$ be a smooth curve over $\mathbb{C}, \tilde{X} \subset T^{*} X$ a smooth spectral curve. To simplify the notation, we assume that $p_{\tilde{X}}: \tilde{X} \rightarrow X$ is ramified at a single point $\tilde{x} \in \tilde{X}$. Denote by $\tilde{X}^{\wedge}$ the formal completion of $\tilde{X}$ at $\tilde{x}$ and by $X^{\wedge}$ the formal completion of $X$ at $x=p_{\tilde{X}}(\tilde{x})$. Clearly, $X^{\wedge}$ is a formal disc and $\tilde{X}^{\wedge}$ is a (smooth ramified) spectral curve over $X^{\wedge}$.

The natural diagram

$$
\begin{aligned}
& \widetilde{\operatorname{Conn}}_{\lambda}(\tilde{X}) \rightarrow \widetilde{\mathcal{C o n n}}_{\lambda}\left(\tilde{X}_{u}\right) \\
& \widetilde{\operatorname{Conn}}_{\lambda}\left(\tilde{X}^{\wedge}\right) \rightarrow \widetilde{\mathcal{C o n n}_{\lambda}}\left(\tilde{X}_{u}^{\wedge}\right)
\end{aligned}
$$

is Cartesian; essentially, the claim is that a $\lambda$-connection on $\tilde{X}$ can be glued from a $\lambda$-connection on $\tilde{X}_{u}$, a $\lambda$-connection on $\tilde{X}^{\wedge}$, and an identification of their restrictions to the punctured disc $\tilde{X}_{u}^{\wedge}:=\tilde{X}_{u} \cap \tilde{X}^{\wedge}$. The same statement holds for $\mathcal{C} \operatorname{con}_{\lambda}(\bullet)$. Now Theorem A follows from Theorem 3.1.

3.2. Let us now prove Theorem 3.1. We will assume that $\tilde{X}$ is ramified over $X=\operatorname{Spf} \mathbb{C}[[z]]$, because the unramified case is simply a 'formal' version of Theorem 2.4 (note also that only the ramified case is used in the proof of Theorem A). It is easy to see that there is a formal coordinate $z$ on $X$ such that $\tilde{X}$ is given by

$$
(\xi-b(z))^{2}=z, \quad(b(z) \in \mathbb{C}[[z]]),
$$

where $\xi$ is the vector field $d / d z$ on $X$. Then $\tilde{X}=\operatorname{Spf} \mathbb{C}[[\tilde{z}]]$ for $\tilde{z}:=\xi-b(z)$.

The following lemma is a version of [9, Theorem 5.2-1] (see also [10]) and can be proved by a similar method:

Lemma 3.2. All objects of $\mathcal{C} \operatorname{onn}_{\lambda}(\tilde{X})$ are isomorphic.

Statement (1) of Theorem A is equivalent to the following claim: 
Proposition 3.3. Suppose $(L, \nabla) \in \mathcal{C} \operatorname{Conn}_{\lambda}(\tilde{X})$; in particular, $L$ is a rank 2 free $\mathbb{C}[[z, \lambda]]$-module. Suppose $L^{\prime} \subset L \otimes_{\mathbb{C}[[z]]} \mathbb{C}((z))$ is a rank 2 free $\mathbb{C}[[z, \lambda]]$-module such that $\nabla\left(L^{\prime}\right) \subset L^{\prime} \otimes \Omega_{X}$. Then $L^{\prime}=L$.

Proof. By Lemma 3.2, we can assume without losing generality that

$$
L=(\mathbb{C}[[z, \lambda]])^{2}, \quad \nabla=\lambda d+b(z) d z+\left(\begin{array}{cc}
0 & z \\
1 & 0
\end{array}\right) d z .
$$

Also by Lemma 3.2 , there exists an isomorphism $(L, \nabla) \rightarrow\left(L^{\prime}, \nabla\right)$. Denote its matrix by

$$
R(z, \lambda)=\left(\begin{array}{ll}
r_{11} & r_{12} \\
r_{21} & r_{22}
\end{array}\right) \in \mathrm{GL}_{2}(\mathbb{C}((z))[[\lambda]]) .
$$

Since the isomorphism is horizontal, we have $[\nabla, R(z, \lambda)]=0$, that is,

$$
\lambda \frac{\partial R(z, \lambda)}{\partial z}=\left[R(z, \lambda),\left(\begin{array}{cc}
0 & z \\
1 & 0
\end{array}\right)\right] .
$$

Now it is easy to see that $R(z, \lambda) \in(\mathbb{C}[[\lambda]])^{\times}$, and therefore $L^{\prime}=L$. Indeed, (3.3) is equivalent to

$$
\begin{aligned}
& \lambda \frac{\partial r_{11}}{\partial z}=-\lambda \frac{\partial r_{22}}{\partial z}=r_{12}-z \cdot r_{21} \\
& \frac{\lambda}{z} \frac{\partial r_{12}}{\partial z}=-\lambda \frac{\partial r_{21}}{\partial z}=r_{11}-r_{22}
\end{aligned}
$$

which implies

$$
\lambda^{2} \frac{\partial^{3} r_{21}}{\partial z^{3}}=4 z \frac{\partial r_{21}}{\partial z}+2 r_{21}
$$

The only solution to (3.4) in $\mathbb{C}((z))[[\lambda]]$ is $r_{21}=0$, which implies that $r_{12}=0$, and that $r_{11}=r_{22}$ does not depend on $z$.

3.3. Statement (2) of Theorem A is proved similarly to (1). Actually, the proof is simpler, because it deals with 'abelian' objects (line bundles).

Lemma 3.4. All objects of $\widetilde{\mathcal{C o n n}}_{\lambda}(\tilde{X})$ are isomorphic.

Proof. Take $\left(l_{1}, \delta_{1}\right),\left(l_{2}, \delta_{2}\right) \in \widetilde{\mathcal{C o n n}}_{\lambda}(\tilde{X})$. Consider the rank 1 free $\mathbb{C}[[\tilde{z}, \lambda]]$ module $l_{1} \otimes\left(l_{2}\right)^{-1}$. It carries a natural $\lambda$-connection, which we denote by $\delta:=$ $\delta_{1} \otimes\left(\delta_{2}\right)^{-1}$. Notice that $\delta$ has no pole (because $\delta_{1}$ and $\delta_{2}$ have equal residues) and that its reduction modulo $\lambda$ is zero (because $\delta_{1}$ and $\delta_{2}$ have equal reductions). Thus, $\lambda^{-1} \delta$ is an ordinary connection on $l_{1} \otimes\left(l_{2}\right)^{-1}$. We can choose a generator $\phi \in l_{1} \otimes\left(l_{2}\right)^{-1}$ such that $\lambda^{-1} \delta(\phi)=0$ (because the formal disc $\tilde{X}$ is simply connected). Such $\phi$ gives an isomorphism $\left(l_{2}, \delta_{2}\right) \widetilde{\rightarrow}\left(l_{1}, \delta_{1}\right)$.

Proposition 3.5. Suppose $(l, \delta) \in \widetilde{\mathcal{C o n n}}_{\lambda}(\tilde{X})$, and suppose $l^{\prime} \subset l \otimes_{\mathbb{C}[[\tilde{z}]]} \mathbb{C}((\tilde{z}))$ is a rank 1 free $\mathbb{C}[[\tilde{z}, \lambda]]$-module such that $\left(l^{\prime}, \delta\right) \in \widetilde{\mathcal{C o n n}_{\lambda}}(\tilde{X})$; in particular, $\delta\left(l^{\prime}\right) \subset \tilde{z}^{-1} l^{\prime} \otimes \Omega_{\tilde{X}}$ (that is, $\delta$ has a first order pole on $l^{\prime}$ at $\left.\tilde{z}=0\right)$. Then $l^{\prime}=l$. 
Proof. By Lemma 3.4, there exists an isomorphism $\iota:(l, \delta) \widetilde{\rightarrow}\left(l^{\prime}, \delta\right)$. Such $\iota$ is given by multiplication by $r(\tilde{z}, \lambda) \in(\mathbb{C}((\tilde{z}))[[\lambda]])^{\times}$. Besides, $[\delta, \iota]=0$, and therefore

$$
\frac{\partial r(\tilde{z}, \lambda)}{\partial \tilde{z}}=0
$$

Hence $r(\tilde{z}, \lambda) \in \mathbb{C}[[\lambda]]^{\times}$and $l^{\prime}=l$.

Proposition 3.5 implies statement (2).

3.4. Now let us prove statement (3). By Lemmas 3.2 and 3.4, both $\left[\mathcal{C}_{\text {onn }}(\tilde{X})\right]$ and $\left[\widetilde{\mathcal{C o n n}}_{\lambda}(\tilde{X})\right]$ are one-element sets. Therefore, it suffices to show that the image of $\left[\widetilde{\mathcal{C o n n}}_{\lambda}(\tilde{X})\right]$ under the bijection $\left[\widetilde{\mathcal{C o n n}}_{\lambda}\left(\tilde{X}_{u}\right)\right] \widetilde{\rightarrow}\left[\mathcal{C o n n}{ }_{\lambda}\left(\tilde{X}_{u}\right)\right]$ is contained in $\left[\mathcal{C o n n}_{\lambda}(\tilde{X})\right]$. This is equivalent to the following statement:

Proposition 3.6. There exist $(L, \nabla) \in \mathcal{C O n n}_{\lambda}(\tilde{X}),(l, \delta) \in \widetilde{\mathcal{C o n n}}_{\lambda}(\tilde{X})$, and a $\mathbb{C}((z))$-linear isomorphism $\phi: L \otimes_{\mathbb{C}[[z]]} \mathbb{C}((z)) \widetilde{\rightarrow} l \otimes_{\mathbb{C}[[\tilde{z}]]} \mathbb{C}((\tilde{z}))$ such that $\phi \circ \nabla=$ $\delta \circ \phi$.

Proof. Define $(L, \nabla)$ by $(3.2)$, and set

$$
l:=\mathbb{C}[[\tilde{z}, \lambda]], \quad \delta:=\lambda d+\left(2 \tilde{z} b\left(\tilde{z}^{2}\right)+2 \tilde{z}^{2}-\frac{\lambda}{2 \tilde{z}}\right) d \tilde{z} .
$$

Clearly, $(l, \delta) \in \widetilde{\mathcal{C o n n}}_{\lambda}(\tilde{X})$. Now define $\phi: L \otimes_{\mathbb{C}[[z]]} \mathbb{C}((z)) \rightarrow l \otimes_{\mathbb{C}[[\tilde{z}]]} \mathbb{C}((\tilde{z}))$ by

$$
\phi(f, g)=\sum_{i=0}^{\infty} \frac{(6 i) !}{(2 i) !(3 i) ! 3^{2 i} 2^{6 i}}\left(\frac{\lambda}{\tilde{z}^{3}}\right)^{i}\left(f-\frac{6 i+1}{6 i-1} \tilde{z} g\right), \quad f, g \in \mathbb{C}((z))[[\lambda]] .
$$

It is easy to see that $\phi$ has the required property.

Remark. The $\lambda$-connection (3.2) is easily reduced to the Airy equation; (3.6) can be obtained from a formal solution to the Airy equation.

\section{Proof of Theorem B}

4.1. Let us start with a simple observation about foliations on formal schemes:

Definition 4.1. A $\lambda$-adic formal scheme is a formal scheme $S$ together with a function $\lambda \in H^{0}\left(S, O_{S}\right)$ such that the zero locus of $\lambda^{i+1}$ is a subscheme $S_{i} \subset S$ and $S=\lim _{\longrightarrow} S_{i}$. A $\lambda$-adic formal scheme $S$ is flat if $S_{i}$ is flat over $\mathbb{C}[\lambda] /\left(\lambda^{i+1}\right)$ for all $i \geq 0$, or, equivalently, if $\lambda \in O_{S}$ is not a zero divisor. Finally, a $\lambda$-adic formal scheme $S$ is smooth if $S_{i}$ is smooth over $\mathbb{C}[\lambda] /\left(\lambda^{i+1}\right)$ for all $i \geq 0$, or, equivalently, if $S$ is flat and $S_{0}$ is smooth over $\mathbb{C}$.

Example 4.2. For an arbitrary $\mathbb{C}$-scheme $S$, set $S[[\lambda]]:=\lim _{\longrightarrow} S \times \operatorname{Spec} \mathbb{C}[\lambda] /\left(\lambda^{i}\right)$ (as in Definition 2.2). Then $S[[\lambda]]$ is a flat $\lambda$-adic formal scheme; it is smooth if and only if $S$ is smooth. 
Lemma 4.3. Let $Y$ and $Z$ be smooth $\lambda$-adic formal schemes, and $\Phi: Y \rightarrow Z$ a morphism over $\mathbb{C}[[\lambda]]$ (that is, $\Phi^{*}(\lambda)=\lambda$ ). Denote by $Y_{0} \subset Y$ and $Z_{0} \subset Z$ the zero loci of $\lambda$.

1. If the restriction of $\Phi$ to $Y_{0}$ is smooth, then so is $\Phi$.

2. Suppose $\Phi$ is smooth, and let $\zeta:=\operatorname{ker}(d \Phi) \subset T Y$ be the foliation corresponding to the fibration $\Phi: Y \rightarrow Z$. Suppose that the quotient $Y_{0} / \zeta$ exists and coincides with $Z_{0}$ (that is, the restriction $\left.\Phi\right|_{Y_{0}}: Y_{0} \rightarrow Z_{0}$ has connected non-empty fibers). Then the quotient $Y / \zeta$ exists and coincides with $Z$.

Our proof of Theorem B is divided into the following steps:

- Construction of a map $\Phi: \mathbf{M}^{\sharp}[[\lambda]] \rightarrow \mathbf{C o n n}_{\text {form }}^{\prime}$.

- Verification that $\Phi$ satisfies the assumptions of Lemma 4.3. Therefore, $\mathbf{C o n n}_{\text {form }}^{\prime}=\mathbf{M}^{\sharp}[[\lambda]] / \zeta$, where the foliation $\zeta$ equals $\operatorname{ker}(d \Phi)$.

- Verification that $\zeta=\zeta_{\lambda}$.

4.2. Construction of $\Phi: \mathbf{M}^{\sharp}[[\lambda]] \rightarrow \mathbf{C o n n}_{\text {form }}^{\prime}$. Even though we formulated Theorems $\mathrm{A}$ and 3.1 for $\mathbb{C}[[\lambda]]$-families of $\lambda$-connections, essentially the same proofs work for $K[[\lambda]]$-families of $\lambda$-connections on a smooth curve $X / K$, where $K$ is an arbitrary $\mathbb{C}$-algebra. Actually, the theorems hold for families parametrized by $S[[\lambda]]$, where $S$ is a $\mathbb{C}$-scheme (or a stack); indeed, the statements are local on $S$.

Recall that $\mathbf{M}^{\sharp}$ is the moduli stack of triples $(\tilde{X}, l, \partial)$ (see Section 2.2); denote by $\left(\tilde{X}^{\sharp}, l^{\sharp}, \partial^{\sharp}\right)$ the universal family on $\mathbf{M}^{\sharp}$. Thus, $\tilde{X}^{\sharp} \subset\left(T^{*} X\right) \times \mathbf{M}^{\sharp}$ is an $\mathbf{M}^{\sharp}$ family of smooth spectral curves, $l^{\sharp}$ is a line bundle on $\tilde{X}^{\sharp}$, and $\partial^{\sharp}: l^{\sharp} \rightarrow$ $l^{\sharp} \otimes \Omega_{\tilde{X}^{\sharp} / \mathbf{M}^{\sharp}}\left(D_{r}\right)$ is a connections with pole at $D_{r}$, the ramification divisor of the projection $\tilde{X}^{\sharp} \rightarrow X \times \mathbf{M}^{\sharp}$. The residue of $\partial^{\sharp}$ at $D_{r}$ equals $-1 / 2$.

Let $\mu=\mu_{\tilde{X}^{\sharp}} \in H^{0}\left(\tilde{X}^{\sharp}, \Omega_{\tilde{X}^{\sharp} / \mathbf{M}^{\sharp}}\right)$ be the natural relative 1 -form on $\tilde{X}^{\sharp}$; it is the pull-back of the natural 1 -form on $T^{*} X$ under the projection $\tilde{X}^{\sharp} \rightarrow T^{*} X$. Denote by $l^{\sharp}[[\lambda]]$ the pull-back of $l^{\sharp}$ to $\tilde{X}^{\sharp}[[\lambda]]$. The expression $\mu+\lambda \partial^{\sharp}$ gives a $\lambda$-connection on $l \sharp[[\lambda]]$ :

$$
\mu+\lambda \partial^{\sharp}: l^{\sharp}[[\lambda]] \rightarrow l^{\sharp}[[\lambda]] \otimes \Omega_{\tilde{X}^{\sharp}[[\lambda]] / \mathbf{M}^{\sharp}[[\lambda]]}\left(D_{r}[[\lambda]]\right) .
$$

So we see that $\mathbf{M}^{\sharp}[[\lambda]]$ carries a natural family of spectral curves $\left(\tilde{X}^{\sharp}[[\lambda]]\right)$ and line bundles with $\lambda$-connections $\left(l^{\sharp}[[\lambda]]\right.$ and $\left.\mu+\lambda \partial^{\sharp}\right)$ on these curves. According to the generalized Theorem A, such a family corresponds to a $\mathrm{GL}_{2}$-bundle $L$ on $\left(\mathbf{M}^{\sharp} \times X\right)[[\lambda]]$ equipped with a $\lambda$-connection $L \rightarrow L \otimes \Omega_{X}$. Such a pair $(L, \nabla)$ gives a map $\Phi: \mathbf{M}^{\sharp}[[\lambda]] \rightarrow$ Conn $_{\lambda}$. Clearly, $\Phi\left(\mathbf{M}^{\sharp}[[\lambda]]\right)$ is contained in $\mathbf{C o n n}_{\text {form }}^{\prime}$ (the formal completion of $\mathbf{C o n n}_{\lambda}$ along $\mathbf{H i g g s}^{\prime}$ ).

It is easy to see that Lemma $4.3(2)$ applies to $\Phi: \mathbf{M}^{\sharp}[[\lambda]] \rightarrow \mathbf{C o n n}_{\lambda}$. Indeed, $\mathbf{M}^{\sharp}$ is smooth, and the map

$$
\lambda: \operatorname{Conn}_{\lambda} \rightarrow \mathbb{C}:(L, \nabla, \lambda) \mapsto \lambda
$$


is smooth on Higgs ${ }^{\prime} \subset \mathbf{C o n n}_{\lambda}$; therefore, both $\mathbf{M}^{\sharp}\left[[\lambda]\right.$ and $\mathbf{C o n n}_{\text {form }}^{\prime}$ are smooth $\lambda$-adic formal stacks. Besides, the restriction of $\Phi$ to $\mathbf{M}^{\sharp} \subset \mathbf{M}^{\sharp}[[\lambda]]$ (the zero locus of $\lambda$ ) is the natural projection

$$
\mathbf{M}^{\sharp} \rightarrow \mathbf{M}^{\sharp} / \zeta_{0}=\text { Higgs' }^{\prime}
$$

4.3. Now let us verify that $\zeta_{\lambda}=\zeta:=\operatorname{ker}(d \Phi)$. As $\operatorname{rk}\left(\zeta_{\lambda}\right)=\operatorname{rk}(\zeta)$, it suffices to check that $\zeta_{\lambda} \subset \zeta$. Equivalently, for an open set $U \subset \mathbf{M}^{\sharp}[[\lambda]]$ and a vector field $\theta$ on $U$ that belongs to $\zeta_{\lambda}$, we need to check that $\theta$ belongs to $\zeta$.

Set $U[\epsilon]:=U \times \operatorname{Spec} \mathbb{C}[\epsilon] /\left(\epsilon^{2}\right)$. The vector field $\theta$ induces an automorphism $\Theta: U[\epsilon] \stackrel{\rightarrow}{\rightarrow}[\epsilon]$ characterized by the following property:

$$
\Theta^{*}(f+\epsilon g)=f+\epsilon(g+\theta(f)) \quad\left(\text { here } f, g \in O_{U}, \text { so } f+\epsilon g \in O_{U[\epsilon]}\right) \text {. }
$$

We need to verify that the two compositions

$$
\Phi \circ \pi, \Phi \circ \pi \circ \Theta: U[\epsilon] \rightarrow \mathbf{C o n n}_{\lambda}
$$

coincide. Here $\pi: U[\epsilon] \rightarrow U \hookrightarrow \mathbf{M}^{\sharp}[[\lambda]]$ is the projection.

Let $\left(\tilde{X}_{1}, l_{1}, \delta_{1}\right)$ and $\left(\tilde{X}_{2}, l_{2}, \delta_{2}\right)$ be the pull-backs of the family $\left(\tilde{X}^{\sharp}[[\lambda]], l \sharp[[\lambda]]\right.$, $\left.\mu+\lambda \partial^{\sharp}\right)$ under $\pi: U[\epsilon] \rightarrow \mathbf{M}^{\sharp}[[\lambda]]$ and $\pi \circ \Theta: U[\epsilon] \rightarrow \mathbf{M}^{\sharp}[[\lambda]]$, respectively. Thus, $\tilde{X}_{i} \subset T^{*} X \times U[\epsilon]$ is a $U[\epsilon]$-family of smooth spectral curves, $l_{i}$ is a line bundle on $\tilde{X}_{i}$, and $\delta_{i}$ is a $\lambda$-connection on $l_{i}$ with the usual condition on the residues $(i=1,2)$. We need to verify that $\left(\tilde{X}_{1}, l_{1}, \delta_{1}\right)$ and $\left(\tilde{X}_{2}, l_{2}, \delta_{2}\right)$ define the same $U[\epsilon]$-family of $\mathrm{GL}_{2}$-bundles with $\lambda$-connections on $X$.

According to Theorem A (or rather its generalized version), it suffices to check the following two statements:

1. The reductions of $\left(\tilde{X}_{1}, l_{1}, \delta_{1}\right)$ and $\left(\tilde{X}_{2}, l_{2}, \delta_{2}\right)$ modulo $\lambda$ coincide.

2. Let $\tilde{X}_{i u} \subset \tilde{X}_{i}$ be the open set where $p_{\tilde{X}_{i}}: \tilde{X}_{i} \rightarrow U[\epsilon] \times X$ is unramified $(i=$ $1,2)$. Then the push-forwards $\left(p_{\tilde{X}_{1}}\right)_{*}\left(\left.\left(l_{1}, \delta_{1}\right)\right|_{\tilde{X}_{1 u}}\right)$ and $\left(p_{\tilde{X}_{2}}\right)_{*}\left(\left.\left(l_{2}, \delta_{2}\right)\right|_{\tilde{X}_{2 u}}\right)$ are canonically isomorphic. Notice that the previous statement implies $p_{\tilde{X}_{1}}\left(\tilde{X}_{1 u}\right)=p_{\tilde{X}_{2}}\left(\tilde{X}_{2 u}\right)$; therefore, these push-forwards are $\mathrm{GL}_{2}$-bundles with $\lambda$-connections on the same open subset of $X \times U[\epsilon]$.

Both statements easily follow from the definition of $\zeta_{\lambda}$.

This completes the proof of statements (2) and (3) of Theorem B. To prove Theorem $\mathrm{B}(1)$, we need to show that the distribution $\zeta_{\lambda} \subset T \mathbf{M}^{\sharp}$ is a foliation, that is, that its curvature

$$
\kappa: \zeta_{\lambda} \otimes \zeta_{\lambda} \rightarrow T \mathbf{M}^{\sharp} / \zeta_{\lambda}: \theta_{1} \otimes \theta_{2} \mapsto\left[\theta_{1}, \theta_{2}\right]
$$

vanishes. However, $\kappa$ depends on $\lambda$ algebraically, and Theorem $\mathrm{B}(2)$ implies that $\kappa=0$ when $\lambda \in \mathbb{C}[[\lambda]]$ is a formal parameter. Theorem $\mathrm{B}(1)$ follows.

\section{5. $\lambda$-connections and Higgs bundles}

5.1. Recall the description of Higgs bundles in terms of spectral curves ([5], see also [3] for a more general statement).

Theorem 5.1. Let $(L, \nabla)$ be a Higgs bundle. 
1. There exists a unique spectral curve $\tilde{X} \in \mathrm{SCurv}$ and a unique (up to a canonical isomorphism) coherent $O_{\tilde{X}}$-module $l$ such that $L=\left(p_{\tilde{X}}\right)_{*} l$ and $\nabla=\left(p_{\tilde{X}}\right)_{*} \mu$. We call $(\tilde{X}, l)$ the spectral data of $(L, \nabla)$.

2. If $\tilde{X}$ is smooth, $l$ is an invertible sheaf on $\tilde{X}$.

3. For a smooth spectral curve $\tilde{X}$ and an invertible sheaf $l$ on $\tilde{X}$, there is a unique (up to a canonical isomorphism) Higgs bundle $(L, \nabla)$ such that $(\tilde{X}, l)$ is the spectral data of $(L, \nabla)$.

Corollary 5.2. Fix a smooth spectral curve $\tilde{X} \in \mathrm{SCurv}$, and let $\mathcal{H}$ iggs $(\tilde{X})$ (resp. $\widetilde{\mathcal{H} i g g s}(\tilde{X}))$ be the groupoid of Higgs bundles $(L, \nabla)$ on $X$ with spectral curve $\tilde{X}$ (resp. the groupoid of line bundles $l$ on $\tilde{X}$ ). Then the functor

$$
l \mapsto\left(\left(p_{\tilde{X}}\right)_{*} l,\left(p_{\tilde{X}}\right)_{*} \mu\right)
$$

is an equivalence $\mathcal{F}_{0}: \widetilde{\mathcal{H} \text { iggs }}(\tilde{X}) \widetilde{\rightarrow}$ iggs $(\tilde{X})$.

5.2. Let us now show that our description of $\lambda$-connections via spectral curves (Corollary 2.5) agrees with the description of Higgs bundles (Corollary 5.2).

Proposition 5.3. The equivalences $\mathcal{F}$ and $\mathcal{F}_{0}$ (see Corollaries 2.5, 5.2) fit into a commutative diagram

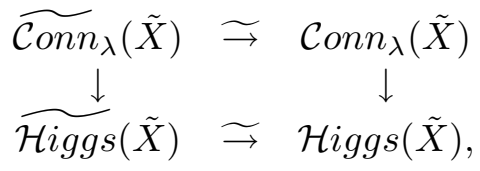

in which the vertical arrows are

$$
\begin{aligned}
& \tilde{\mathcal{R}}: \widetilde{\mathcal{C o n n}}_{\lambda}(\tilde{X}) \rightarrow \widetilde{\mathcal{H i g g s}}(\tilde{X}):(l, \delta) \mapsto l / \lambda l, \\
& \mathcal{R}: \mathcal{C o n n}_{\lambda}(\tilde{X}) \rightarrow \mathcal{H i g g s}(\tilde{X}):(L, \nabla) \mapsto\left(L_{0}, \nabla_{0}\right) .
\end{aligned}
$$

Here $\left(L_{0}, \nabla_{0}\right)$ is the reduction of $(L, \nabla)$ modulo $\lambda$.

Proof. Take any $(l, \delta) \in \widetilde{\mathcal{C O n n}_{\lambda}}(\tilde{X})$, and set $(L, \nabla):=\mathcal{F}(l, \delta),\left(L_{0}, \nabla_{0}\right):=$ $\mathcal{R}(L, \nabla),\left(L_{0}^{\prime}, \nabla_{0}^{\prime}\right):=\mathcal{F}_{0} \circ \tilde{\mathcal{R}}(l, \delta)$. We need to construct an isomorphism between the Higgs bundles $\left(L_{0}^{\prime}, \nabla_{0}^{\prime}\right)$ and $\left(L_{0}, \nabla_{0}\right)$.

By definition of $\mathcal{F}$, there exists an isomorphism

$$
\phi:\left.p_{\tilde{X}}\left(\left.(l, \delta)\right|_{\tilde{X}_{u}}\right) \widetilde{\rightarrow}(L, \nabla)\right|_{X_{u}} .
$$

It induces an isomorphism

$$
\phi_{0}:\left.L_{0}^{\prime}\right|_{X_{u}}=\left.p_{\tilde{X}}\left(\left.(l / \lambda l)\right|_{\tilde{X}_{u}}\right) \widetilde{\rightarrow}(L / \lambda L)\right|_{X_{u}}=\left.L_{0}\right|_{X_{u}} .
$$

Clearly, $\phi_{0} \circ \nabla_{0}^{\prime}=\nabla_{0} \circ \phi_{0}$, because $\phi$ agrees with the $\lambda$-connections. The proposition now follows from Lemma 5.4.

Lemma 5.4. $\phi_{0}$ extends to an isomorphism between $L_{0}^{\prime}=p_{\tilde{X}}(l / \lambda l)$ and $L_{0}=$ $L / \lambda L$. 
Proof. It suffices to consider that case when $X=\operatorname{Spf} \mathbb{C}[[z]]$ and $\tilde{X}$ is given by (3.1). $\phi$ is then defined by Proposition 3.6: without loss of generality, we can assume that $(L, \nabla)$ and $(l, \delta)$ are given by (3.2) and (3.5), respectively, in which case $\phi$ is given by (3.6). It is clear from (3.6) that $\phi$ induces a $\mathbb{C}[[z]]$-linear isomorphism $L / \lambda L \stackrel{\sim}{\rightarrow} l / \lambda l$.

\section{Moduli of $\lambda$-connections and the Hitchin fibration}

In this section, we provide a construction that, given a completely integrable system $M \rightarrow B$, produces a formal deformation of the dual abelian scheme $M^{\vee}$. We then show that if $M \rightarrow B$ is the Hitchin integrable system, this deformation 'almost coincides' with Conn $_{\text {form }}^{\prime}$; more precisely, they are isomorphic after an étale base change $\tilde{B} \rightarrow B$.

To simplify the exposition, we avoid algebraic stacks in this section, and work with coarse moduli spaces instead.

6.1. Let $\pi: M \rightarrow B$ be a completely integrable system:

Definition 6.1. A completely integrable system is an abelian scheme $\pi: M \rightarrow B$ over a smooth base $B$ together with a symplectic structure $\omega$ on $M$ such that the fibers of $\pi$ and the image of the zero section $0: B \rightarrow M$ are Lagrangian.

Denote by $M^{\vee}$ the dual abelian scheme, and by $M^{\natural}$ its universal extension. Explicitly, $M^{\vee}$ is the coarse moduli space of pairs $(b, l)$, and $M^{\natural}$ is the coarse moduli space of triples $(b, l, \partial)$, where $b \in B, l$ is a line bundle on the fiber $\pi^{-1}(b)$ with Chern class 0 , and $\partial$ is a flat connection on $l$.

$M^{\natural}$ is equipped with two foliations: $\zeta_{0}$ is the relative tangent bundle to the projection

$$
M^{\natural} \rightarrow M^{\vee}:(b, l, \partial) \mapsto(b, l),
$$

while $\zeta_{\infty}$ is defined using the isomonodromic deformation (similarly to the definition of $\zeta_{\infty}$ in Section 2.2).

As vector bundles on $M^{\natural}$, the foliations $\zeta_{0}$ and $\zeta_{\infty}$ are naturally isomorphic. Indeed, the fiber of $\zeta_{0}$ at $(b, l, \partial)$ is the space of global differential 1-forms on $\pi^{-1}(b) \subset M$, while the fiber of $\zeta_{\infty}$ is the tangent space to $B$ at $b$; the two vector spaces are identified by the symplectic form on $M$. We can therefore form the linear combination $\zeta_{\lambda}:=\zeta_{0}-\lambda \zeta_{\infty}$.

Proposition 6.2. $\zeta_{\lambda}$ is a foliation for any $\lambda \in \mathbb{C}$.

Notice that the quotient $M^{\natural} / \zeta_{0}$ equals $M^{\vee}$. Proposition 6.2 implies that the quotient $M^{\natural} / \zeta_{\lambda}$ exists if $\lambda \in \mathbb{C}[[\lambda]]$ is a formal parameter; the quotient is a canonical formal deformation of $M^{\vee}$.

The proof of Proposition 6.2 will be given elsewhere. However, if $M \rightarrow B$ is the Hitchin fibration (which is the most important case for us), Proposition 6.2 follows from Theorem B(1) and Proposition 6.3. 
6.2. Let $X$ be a smooth projective curve. Denote by $B \subset$ SCurv the space of smooth spectral curves $\tilde{X} \subset T^{*} X$, and let $M$ be the coarse moduli space of pairs $(\tilde{X}, l)$, where $\tilde{X} \in B$, and $l$ is a line bundle on $\tilde{X}$ with Chern class zero. Notice that $M$ is an open subset in the coarse moduli space of Higgs bundles on $X$.

It is well known that $M$ has a symplectic form which turns $M \rightarrow B$ into a completely integrable system: the Hitchin integrable system. Let us apply the above construction to $M \rightarrow B$.

$M \rightarrow B$ is principally polarized, so $M^{\vee}=M$. The universal extension $M^{\natural}$ is identified with the coarse moduli space of triples $(\tilde{X}, l, \partial)$, where $(\tilde{X}, l) \in M$, and $\partial$ is a connection on $l$. The foliation $\zeta_{0}$ is the relative tangent bundle to $M^{\natural} \rightarrow M^{\vee}$, while $\zeta_{\infty}$ is given by the isomonodromic deformation of connections.

Consider now the coarse moduli space corresponding to $\mathbf{M}^{\sharp}$, which we denote by $M^{\sharp}$. Recall that $M^{\sharp}$ is the coarse moduli space of triples $(\tilde{X}, l, \partial)$, where $\tilde{X} \in B \subset$ SCurv, $l$ is a line bundle on $\tilde{X}$, and $\partial$ is a connection on $\tilde{X}$ with simple poles at $\tilde{x}_{1}, \ldots, \tilde{x}_{n} \in \tilde{X}$ (the ramification points of $\tilde{X} \rightarrow X$ ) whose residue at $\tilde{x}_{i}$ equals $-1 / 2$. The space $M^{\sharp}$ also carries natural foliations $\zeta_{0}, \zeta_{\infty}$ defined as above.

Clearly, $M^{\natural} \rightarrow B$ is a group scheme and $M^{\sharp} \rightarrow B$ is a torsor over it. Moreover, the action of $M^{\natural}$ on $M^{\sharp}$ agrees with the foliations $\zeta_{0}, \zeta_{\infty}$ (and so also with foliations $\zeta_{\lambda}, \lambda \in \mathbb{C}$ ). In this sense, the description of the (coarse) moduli space of $\lambda$-connections given by Theorem B is a 'twisted' version of the construction of Section 6.1 applied to the Hitchin integrable system. Here is a slightly stronger statement:

Proposition 6.3. There exists an étale cover $\tilde{B} \rightarrow B$ and an isomorphism

$$
M^{\natural} \times_{B} \tilde{B} \underset{\rightarrow}{\rightarrow} M^{\sharp} \times_{B} \tilde{B}
$$

that preserves the foliations $\zeta_{\infty}$ and $\zeta_{0}$.

Proof. Let $\tilde{B}$ be the coarse moduli space of pairs $(\tilde{X}, \gamma)$, where $\tilde{X} \in B$, and $\gamma$ is a line bundle on $\tilde{X}$ such that

$$
\gamma^{\otimes 2} \simeq O_{\tilde{X}}\left(\tilde{x}_{1}+\cdots+\tilde{x}_{n}\right)=\Omega_{\tilde{X}} \otimes p_{\tilde{X}}^{*}\left(\Omega_{X}\right)^{-1} .
$$

As before, $\tilde{x}_{1}, \ldots, \tilde{x}_{n}$ are the ramifications of $p_{\tilde{X}}: \tilde{X} \rightarrow X$. Clearly, the natural projection $\tilde{B} \rightarrow B$ is an étale cover.

Suppose now $(\tilde{X}, l, \partial, \gamma) \in M^{\natural} \times_{B} \tilde{B}$, that is, $(\tilde{X}, l, \partial) \in M^{\natural}$ and $(\tilde{X}, \gamma) \in$ $\tilde{B}$. Let us also choose an isomorphism (6.1). It induces a connection $\partial_{\gamma}$ on $\gamma$ such that $\left(\tilde{X}, \gamma, \partial_{\gamma}\right) \in M^{\sharp}$. Consider now $\left(\tilde{X}, \gamma \otimes l, \partial_{\gamma} \otimes \partial\right) \in M^{\sharp}$; clearly, its isomorphism class does not depend on the choice of isomorphism (6.1). Define the map $M^{\natural} \times_{B} \tilde{B} \sim M^{\sharp} \times_{B} \tilde{B}$ by

$$
(\tilde{X}, l, \partial, \gamma) \mapsto\left(\tilde{X}, \gamma \otimes l, \partial_{\gamma} \otimes \partial, \gamma\right) .
$$

It is easy to see that it has the required properties. 


\section{Acknowledgments}

I am deeply grateful to V. Drinfeld for his constant attention to this work and for numerous stimulating discussions. Part of this work is contained in the thesis I presented at Harvard University, and I would like to thank my readers, D. Kazhdan and A. Braverman, for their invaluable comments. D. Kazhdan also supervised my studies at Harvard, and I would like to thank him for his guidance.

This work owes much to the discussions I had with many mathematicians. Among them, I am most grateful to A. Beilinson, D. Ben-Zvi, R. Bezrukavnikov, P. Deligne, R. Donagi, D. Gaitsgory, and T. Pantev.

\section{References}

[1] D. Arinkin, Moduli of connections with a small parameter on a curve, Electronic preprint Math. AG/0409373.

[2] - Orthogonality of natural sheaves on moduli stacks of SL(2)-bundles with connections on $\mathbb{P}^{1}$ minus 4 points, Selecta Math. (N.S.), 7, no.2, (2001) 213-239.

[3] R. Y. Donagi and D. Gaitsgory, The gerbe of Higgs bundles, Transform. Groups, 7, no.2, (2002) 109-153.

[4] V. G. Drinfeld and V. V. Sokolov, Lie algebras and equations of Korteweg-de Vries type, (Russian) Current problems in mathematics, Vol. 24, Itogi Nauki i Tekhniki, 81-180. Akad. Nauk SSSR Vsesoyuz. Inst. Nauchn. i Tekhn. Inform., Moscow, 1984.

[5] N. Hitchin, Stable bundles and integrable systems. Duke Math. J., 54, no. 1, (1987) 91114.

[6] C. Simpson, Nonabelian Hodge theory. Proceedings of the International Congress of Mathematicians, Vol. I, II (Kyoto, 1990), 747-756, Math. Soc. Japan, Tokyo, 1991.

[7] _ The Hodge filtration on nonabelian cohomology, In Algebraic geometry-Santa Cruz 1995, Proc. Sympos. Pure Math. 62, 217-281. Amer. Math. Soc., Providence, RI, 1997.

[8] W. Wasow, Asymptotic expansions for ordinary differential equations. Pure and Applied Mathematics, Vol. XIV. Interscience Publishers John Wiley \& Sons, Inc., New YorkLondon-Sydney, 1965.

[9] Linear turning point theory, Applied Mathematical Sciences 54. Springer-Verlag, New York, 1985.

[10] _ Simplification of turning point problems for systems of linear differential equations, Trans. Amer. Math. Soc., 106, (1963) 100-114.

Department of Mathematics, University of Chicago, Chicago, Illinois 60637, USA

E-mail address: arinkin@math.uchicago.edu 